\title{
Step-Tracking Movements of the Wrist in Humans. II. EMG Analysis
}

\author{
Donna S. Hoffman and Peter L. Strick \\ Research Service, V.A. Medical Center and Departments of Neurosurgery and Physiology, SUNY Health Science Center \\ at Syracuse, Syracuse, New York 13210
}

\begin{abstract}
We asked human subjects to make accurate step-tracking movements of the wrist to targets that required $5^{\circ}-30^{\circ}$ of radial or ulnar deviation. Speed instructions were given prior to each trial. Muscle activity was recorded from extensor carpi radialis longus (ECRL) and extensor carpi ulnaris (ECU) using surface electrodes. The agonist muscle initiated each movement with a brief burst of activity which began approximately $45 \mathrm{msec}$ before movement onset. Then, the antagonist muscle displayed a brief burst of activity which began approximately $10 \mathrm{msec}$ after movement onset. The magnitude, but not the timing, of these bursts was modulated by changes in the task requirements.
\end{abstract}

The area of the initial agonist burst varied with changes in both displacement and intended speed. This burst was most highly correlated with the initial peaks of acceleration and jerk. In contrast, the area of the initial antagonist burst varied with changes in intended speed and was less well modulated by changes in displacement. This burst was highly correlated with the reciprocal of movement duration. Some small, fast movements had the same agonist bursts as some large, slow movements. However, the antagonist bursts for these movements differed greatly. This observation provides clear evidence that the magnitudes of the agonist and antagonist bursts are independently controlled.

In a prior paper (Hoffman and Strick, 1986b), we proposed that step-tracking movements of different amplitudes and intended speeds are centrally generated by adjusting 2 kinematic variables: (1) the peak value and (2) the duration of a derivative of displacement. The present results suggest that these $\mathbf{2}$ kinematic parameters are separately generated by independently modulating the magnitudes of the agonist and antagonist bursts. Thus, the peak displacement of a step-tracking movement must be determined by the appropriate adjustment of both bursts of muscle activity.

This paper is part of a series of studies on the control of steptracking movements of the wrist in humans (Waters and Strick, 1981; Hoffman and Strick, 1986a, b). Step-tracking movements have been the focus of recent studies, in part, because these movements are thought to be largely preprogrammed by the CNS and only modulated by peripheral feedback (e.g., Hallett ct al., 1975; Wachholder and Altenburger, 1926; Rothwell et

\footnotetext{
Received Sept. 14, 1988; revised May 31, 1989; Accepted June 29, 1989.

This work has been supported in part through funds from the Veterans Administration Medical Research Service and the Department of Neurosurgery. We thank Mark Lowery and Lco Walsh for their expert technical assistance.

Correspondence should be addressed to Dr. Peter L. Strick, Research Service (151) V.A. Medical Center, Syracuse, NY 13210.

Copyright (c) 1990 Society for Neuroscience $0270-6474 / 90 / 010142-11 \$ 02.00 / 0$
}

al., 1982). Thus, considerable insight into the central control of movement might come from an analysis of the patterns of muscle activity which generate step-tracking movements and how these patterns are adjusted to meet different task requirements.

Step-tracking movements are produced by a complex, but well-defined pattern of muscle activity (e.g., Wachholder and Altenburger, 1926; Hallett et al., 1975; Hallett and Marsden, 1979; Lestienne, 1979; Brown and Cooke, 1981). This pattern is characterized by distinct bursts and pauses in the activity of agonist and antagonist muscles. Agonist muscles initiate steptracking movements with a short burst of activity and then become relatively inactive during the initial trajectory of the movement. Generally, while the agonist muscles are inactive, antagonist muscles display a burst of activity. This burst begins at or just after movement onset. In many instances, additional bursts of muscle activity alternate in agonist and antagonist muscles until the limb is stabilized in the target zone.

Prior studies have examined the modulations in the initial agonist and antagonist bursts while subjects performed different types of step-tracking movements. In some experiments, the magnitudes of the initial agonist and antagonist bursts varied with limb displacement and/or limb velocity (Hallett and Marsden, 1979; Lestienne, 1979; Brown and Cooke, 1981; Waters and Strick, 1981; Marsden et al., 1983; Karst and Hasan, 1987). In other experiments, the magnitude of the antagonist burst was independent of limb displacement (Hallett and Marsden, 1979; Brown and Cooke, 1981). Taken together, the results of prior studies do not provide a clear consensus about how the two bursts relate to different kinematic variables.

In a companion study, we examined the kinematics of steptracking movements of the wrist (Hoffman and Strick, 1986b). Based on the results of that study we proposed that movements of different amplitudes and intended speeds are centrally generated by adjusting 2 kinematic parameters: (1) the peak value and (2) the duration of a derivative of displacement. In the present study, we have examined the relationships between the initial agonist and antagonist bursts and various kinematic parameters. We reasoned that strong relationships between muscle activity and movement parameters would provide insights into the movement parameters generated by each burst of muscle activity and, thus, the parameters controlled by the CNS. Our results suggest that the 2 kincmatic parameters which we proposed as important for the central control of step-tracking movements are generated by separate bursts of muscle activity. Furthermore, our results indicate that the appropriate adjustment of the initial agonist and antagonist bursts of muscle activity is essential for specifying the peak displacement of a step-tracking movement. Abstracts of some of these data have been presented previously (Hoffman and Strick, 1981, 1982, 1984). 


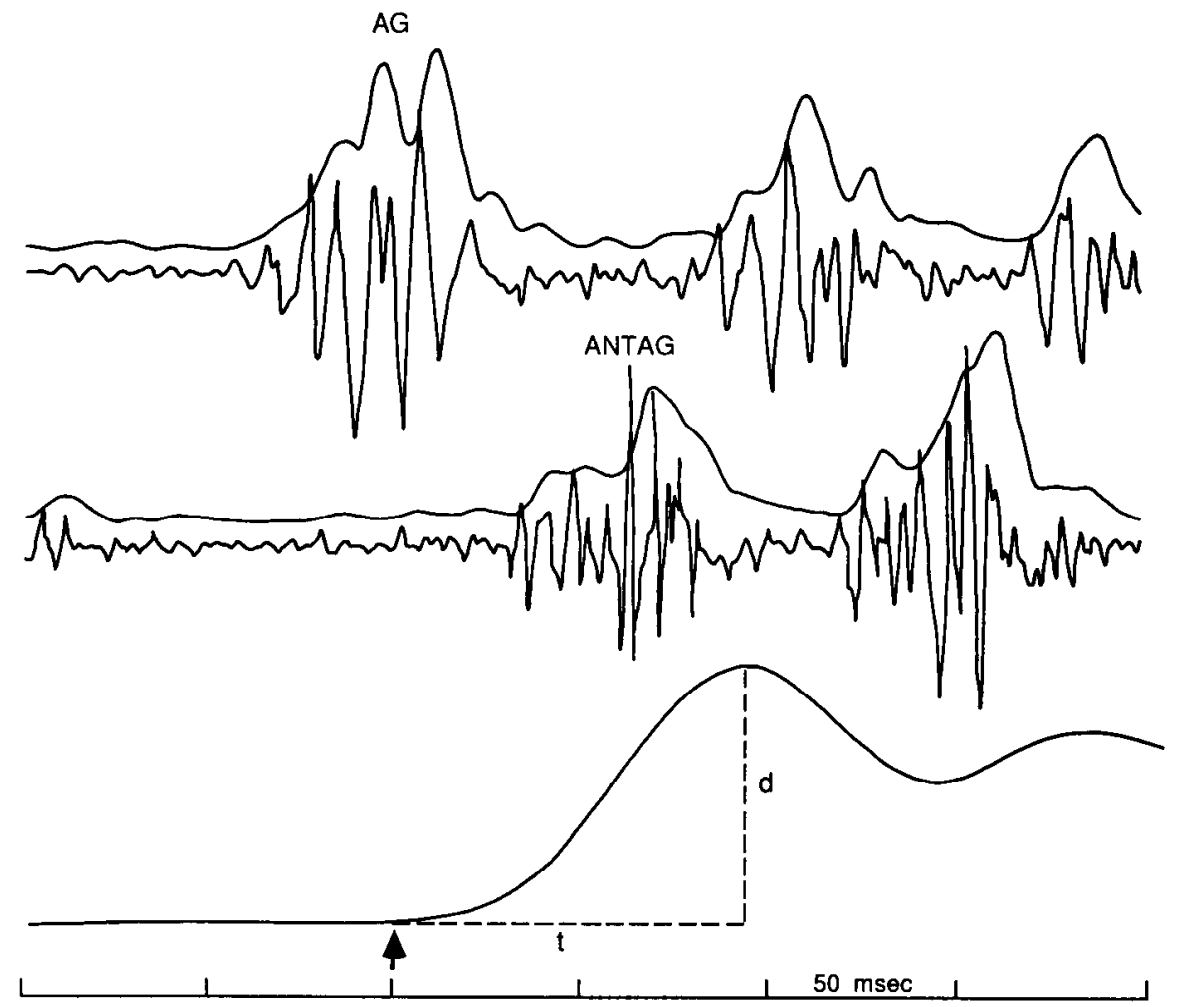

Figure 1. A single step-tracking movement associated with bursts of activity in agonist and antagonist muscles. Top. Agonist muscle activity was recorded from extensor carpi radialis longus. Only the initial agonist burst is labeled $(A G)$. The smooth continuous lines in the top and middle portions of the figure show muscle activity after it has been full-wave rectified and filtered (Gottlieb and Agarwal, 1970; $\tau=10$ $\mathrm{msec}$ ). Middle, Antagonist muscle activity was recorded from extensor carpi ulnaris. Only the initial antagonist burst is labeled $(A N T A G)$. Bottom, Wrist displacement for a single movement. The subject was instructed to move "as accurately and as fast as possible" to a target which required radial deviation of the wrist. Movement onset is indicated by the large solid arrow. The peak of the initial trajectory is indicated by the dashed line $(d)$. The movement duration is indicated by the dashed line $(t)$

\section{Materials and Methods}

\section{Experimental setup}

We examined the patterns of muscle activity of 5 human subjects (aged 25-38; with no hislory of neurological abnormalities). The kinematics of the movements of these subjects were reported in a previous study (Hoffman and Strick, 1986b). Two of the subjects were studied in multiple sessions using multiple paradigms.

Each subject sat in a chair with the forearm and elbow of the dominant limb supported. The subject grasped the handle of a manipulandum which allowed the wrist to rotate freely. Two potentiometers were coupled to the device and measured the angle of the wrist in the planes of flexion-extension and ulnar-radial deviation. A photograph of the manipulandum and further details about its design have been presented previously (see figure 1, Hoffman and Strick, 1986b).

The subject sat approximately $70 \mathrm{~cm}$ in front of a large screen oscilloscope $(26 \times 36 \mathrm{~cm})$ that displayed a cursor and a target. The cursor was a small spot of light (approximately $5 \times 5 \mathrm{~mm}$ ) that moved in proportion to the subject's wrist movements. One degree of wrist movement moved the cursor $4.5 \mathrm{~mm}$ on the screen. For a right-handed subject, the cursor moved right for extension, left for flexion, up for radial deviation, and down for ulnar deviation of the wrist. The target was, in all cases, an open square whose inside diameter equaled $2.5^{\circ}$ of wrist movement. It indicated where the subject should place the cursor. The location of the target on the screen was determined by a computer (DEC 11/34) that was programmed to alter target location according to task requirements.

\section{Task}

The task performed by subjects in this experiment has been described previously (Hoffman and Strick, 1986b). Each subject was asked to perform a step-tracking task which required radial or ulnar deviation of the wrist. In order to perform these movements, each subject had to prevent flexion or extension since the handle could move easily in all directions. When movements were made in the radial direction, the initial position of the target required $10^{\circ}$ of ulnar deviation. When movements were made in the ulnar direction, the initial position of the target required $20^{\circ}$ of radial deviation. Since the experimental paradigms described below required large numbers of trials, subjects were given frequent rest periods and were observed for possible signs of fatigue.

In the first series of trials for each experimental session, the subject was instructed to perform movements "as fast as possible." Target jumps required $5^{\circ}, 10^{\circ}, 15^{\circ}, 20^{\circ}, 25^{\circ}$, and $30^{\circ}$ changes in wrist angle. The same target location was presented for a block of 25-50 trials. The order in which the different target locations was presented was randomized.

Four of the five subjects were asked to perform a second series of trials. For these trials, the subject was given 1 of 3 different speed instructions: "move as fast as possible," "move at your natural speed," and "move at half your natural speed." We will use the term intended speed to indicate the subject's interpretation of these instructions. Target jumps required $10^{\circ}$ and $20^{\circ}$ changes in wrist angle. The same displacement and speed instructions were presented for a block of 30-50 trials.

One subject was reexamined in a separate experimental session using a different protocol. The subject was asked to perform about 30 movements as fast as possible to a target. Then the subject was asked to move to the same target while the speed instruction was randomly varied. Instructions were: "move a little slower," "move a little faster," and "move at the same speed." The target jumps required $5^{\circ}, 15^{\circ}$, and $25^{\circ}$ changes in wrist angle. The same target was presented for a block of 150 trials.

\section{Data acquisition}

Electromyographic (EMG) activity was recorded with Beckman pediatric EMG electrodes. Electrode pairs were spaced about $1.0 \mathrm{~cm}$ apart on the skin overlying the extensor carpi radialis longus (ECRL) and the extensor carpi ulnaris (ECU). The position of the electrodes was carefully adjusted until we recorded little or no activity with finger movements and large responses with wrist movements.

The raw EMG signals were amplified and filtered using standard techniques. These signals were monitored on a storage oscilloscope during the experiment and were tape-recorded for later analysis. Signals from the position transducers and an analog code representing events related to the task were tape-recorded simultancously. Later, the taperecorded EMG signals were full-wave rectified and filtered (Fig. 1, $\tau=$ $10 \mathrm{msec}$; see Gottlieb and Agarwal, 1970). A DEC PDP $11 / 34$ computer was used off-line to digitize the signals from individual trials at $2.5 \mathrm{kHz}$. 
Figure 2. Kinematics and muscle activity for step-tracking movements performed as fast as possible. Solid lines, Averages of 40 movements to the $25^{\circ}$ larget; dot-dashed lines, averages of 42 movements to the $15^{\circ}$ target; dashed lines, averages of 27 movements to the $5^{\circ}$ target. The subject performed movements in the radial direction starting from an initial position of $10^{\circ}$ ulnar deviation. $A$ and $B$, Displacement and acceleration for these movements have been modified from Figure 3 in Hoffman and Strick (1986b). $C$, Agonist muscle activity was recorded from extensor carpi radialis longus. $D$, Antagonist muscle activity was recorded from extensor carpi ulnaris. Note that although the magnitude of agonist and antagonist activity varied for movements of different amplitude, the time course of the bursts of muscle activity remained relatively constant.
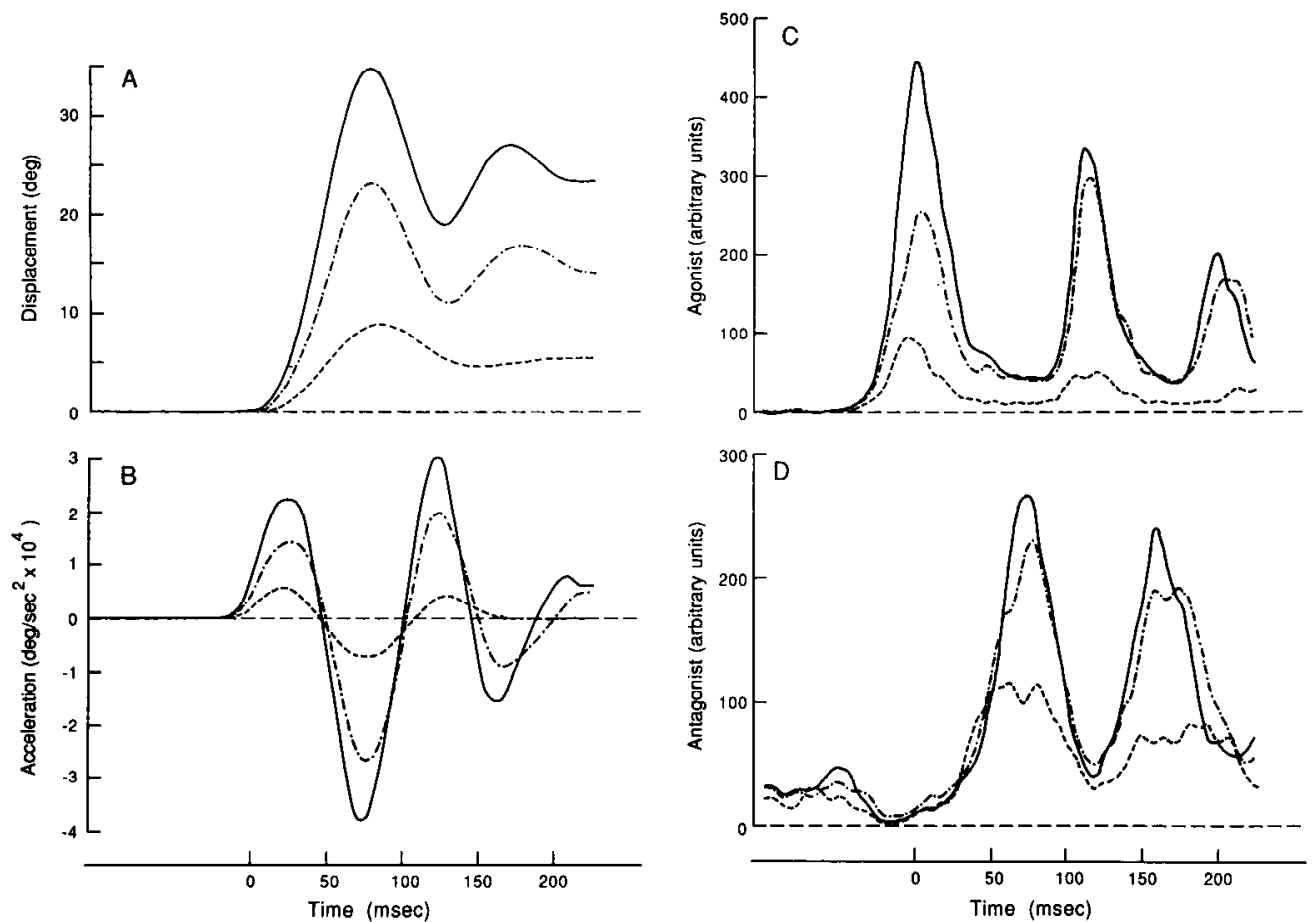

\section{Data analysis}

$E M G$. The presence of bursts of muscle activity was determined by visual inspection of data from single trials. The timing of these bursts was determined from visual inspection of averages of trials aligned on movement onset. The baseline for our measurements of muscle activity was the average EMG activity in the $20 \mathrm{msec}$ interval which immediately preceded each burst of muscle activity. Although we measured the peak and area of the initial agonist and antagonist bursts for each trial, our results will report only the data for area. This is because our initial analysis showed that the area of these bursts was better correlated with kinematic variables than peak activity. The times of onset of EMG activity in relation to movement differed among subjects. Therefore, analysis intervals were determined separately for each subject. Agonist burst area was measured over an $80 \mathrm{msec}$ time period that began approximately $40 \mathrm{msec}$ before movement onset. Antagonist burst area was measured over a $90 \mathrm{msec}$ time period that began approximately $20 \mathrm{msec}$ after movement onset.

Kinematics. The digitized potentiometer signals were low-pass-filtered by the computer (cutoff frequency $=200 \mathrm{~Hz}$ ). Filtering was applied in the backward as well as the forward direction in order to correct for phase shifts. Filtered signals were then successively differentiated to derive velocity, acceleration, and jerk (change in acceleration). The following variables were determined for the initial trajectory of each movement: movement duration (Fig. 1,t) and the peaks of displacement (Fig. $1, d)$, velocity, acceleration, and jerk. Movement duration was defined as the time from movement onset to the first peak in displacement (i.e., the time between movement onset and the next zero value of velocity).

\section{Results}

Regardless of intended speed, the initial trajectory of step-tracking movements of the wrist was characterized by a rapid, but smooth change in position (Figs. 1, 2A, 4A). During this period, each of the derivatives of displacement also changed smoothly and had only one positive peak (Figs. $2 B, 4 B$ ). Thus, the initial movement trajectory was performed in a single "step" rather than in multiple, smaller steps. Movement was terminated after a number of oscillations about the target. The preceding paper in this series reported the results of our examination of the kinematics of these movements in detail (Hoffman and Strick, 1986b).
A well-defined pattern of activity was present in the agonist and the antagonist muscles during step-tracking movements of the wrist (Fig. 1). The agonist muscle initiated each movement with a burst of activity (Figs. $2 C, 4 C$ ). In contrast, the antagonist muscle decreased its activity prior to movement onset and then had a burst of activity which followed movement onset (Figs. $2 D, 4 D)$. Alternating bursts $(n=2-8)$ in agonist and antagonist muscles also occurred during later stages of the movement. This pattern of agonist and antagonist activity was present for movements with durations as long as $218 \mathrm{msec}$ and as short as 58 msec. Similar patterns of activity have been described for agonist and antagonist muscles during step-tracking movements at other joints (e.g., Wachholder and Altenburger, 1926; Hallett et al., 1975; Hallett and Marsden, 1979; Lestienne, 1979; Wadman et al., 1979; Brown and Cooke, 1981; Ghez and Martin, 1982). The present report will focus on the relationships between the initial agonist and antagonist bursts and kinematic parameters related to the initial trajectory of step-tracking movements.

\section{Movements performed as fast as possible}

\section{Agonist amplitude}

When subjects performed step-tracking movements as fast as possible, changes in movement amplitude were associated with large alterations in both the peak and the area of the agonist burst, but not the duration of the burst (Fig. 2C). In fact, the area of the agonist burst displayed high correlations $(r=0.80-$ 0.92 ) with the initial peaks of displacement, velocity, acceleration, and jerk. For individual subjects, the correlations between the area of the agonist burst and each of the initial kinematic parameters did not significantly differ from one another. This is not surprising since the peaks of displacement and its derivatives were all highly correlated (Hoffman and Strick, 1986b). An example of the relation between the area of the agonist burst and peak acceleration is shown for the movements of one subject in Figure $3 A$. Note that the linear regression line has a $y$-intercept that is statistically indistinguishable from zero. This feature was 


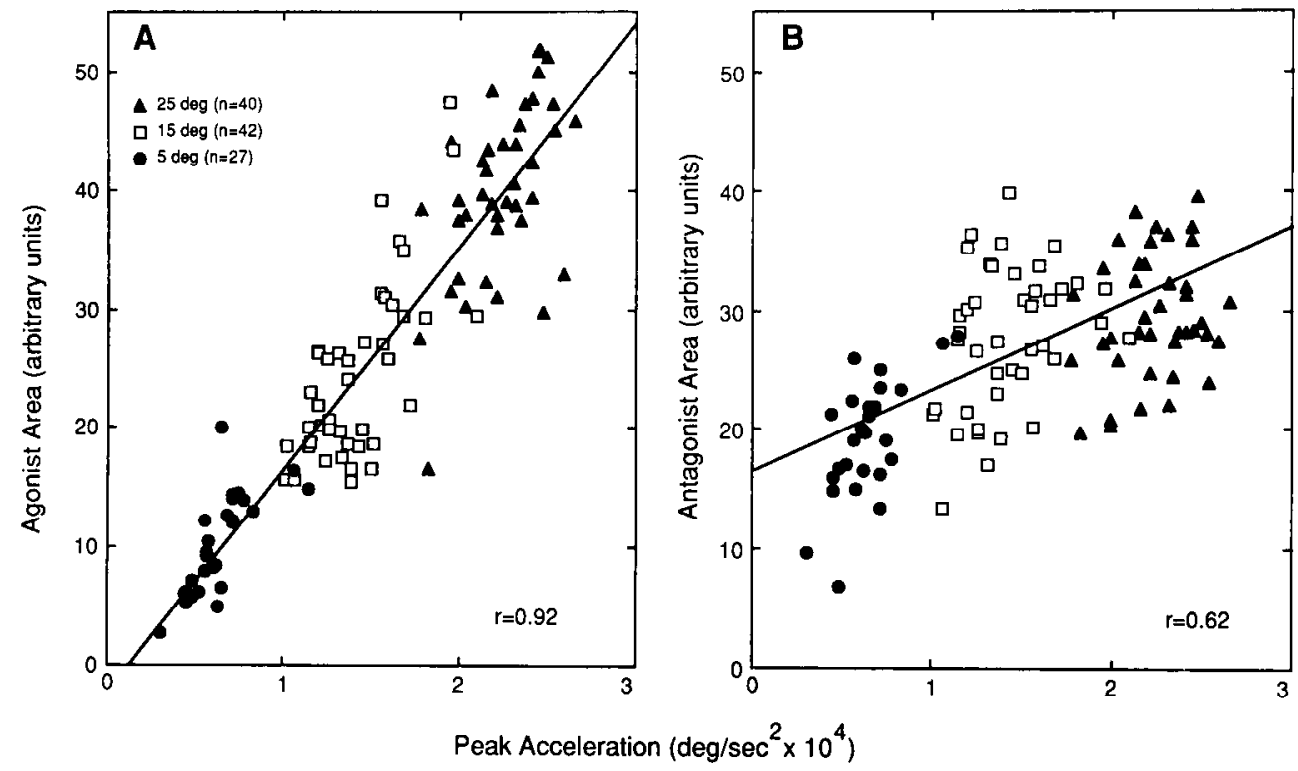

Figure 3. Relationships between acceleration and the initial agonist and antagonist bursts of muscle activity. The data and the linear regression lincs arc from individual trials of one subject performing movements as fast as possible. Targets required changes in wrist angle of $5^{\circ}, 15^{\circ}$, and $25^{\circ}$ in the radial direction. $A$, Agonist muscle activity recorded from extensor carpi radialis longus. $B$, Antagonist muscle activity recorded from extensor carpi ulnaris. The averages of these trials are illustrated in Figure 2. also present in the graphs relating the area of the agonist burst to the peaks of displacement, velocity, and jerk (not illustrated). Thus, for movements performed as fast as possible, the area of the agonist burst was directly proportional to the peak of displacement and to the peaks of the derivatives of displacement.

\section{Antagonist amplitude}

The initial antagonist burst was a substantial burst of muscle activity (Figs. 1, 2D, 4D). Its amplitude could be as large as the initial agonist burst in the same muscle for movements in the opposite direction. Changes in movement amplitude were associated with alterations in the peak and area of the antagonist burst, but not its duration (Figs. $2 D, 3 B$ ). Larger alterations in this burst were associated with changes in intended speed (see below and Figs. $4 D ; 5, C, D$ ). For movements performed as fast as possible, the correlations between the area of the antagonist burst and the initial peaks of displacement, velocity, acceleration, and jerk, as well as those with deceleration and negative jerk, varied greatly between subjects $(r=0.29-0.73)$. However, for any one subject the correlations between the area of the antagonist burst and each of the kinematic variables did not differ significantly from one another.

An example of one of the best relationships between a kinematic variable (in this case, peak acceleration) and the antagonist burst during movements performed as fast as possible is shown in Figure $3 B$ (the agonist activity for the same movements was plotted in Fig. $3 A$ ). Note that the linear regression line for this graph has a large $y$-intercept. This was also observed in the graphs relating the area of the antagonist burst and the peak of displacement or the peaks of the other derivatives. Furthermore, the antagonist bursts for movements to the $15^{\circ}$ and $25^{\circ}$ targets differed by only a small amount, even though the antagonist bursts for movements to the $15^{\circ}$ target were nearly double those for movements to the $5^{\circ}$ target (Fig. $3 B$ ). These observations illustrate that, at best, the relationship between the antagonist burst and peak acceleration was nonlinear. Clearly, the magnitude of the antagonist burst in a step-tracking movement is not tightly coupled to the initial peaks of displacement and its derivatives.

\section{Agonist timing}

The onset and duration of the agonist burst in ECRL and ECU were generally constant for different amplitude movements performed as fast as possible (Fig. 2). The burst began an average of $46 \mathrm{msec}$ prior to movement onset and had an average duration of $97 \mathrm{msec}$. In 2 of the 5 subjects we examined, the duration of the agonist burst for large-amplitude movements $\left(25^{\circ}\right)$ was approximately $22 \mathrm{msec}$ longer than the burst duration for small-amplitude movements $\left(5^{\circ}\right)$. In both subjects, the increase in burst duration occurred only for movements in 1 of the 2 directions. Thus, in most instances, large changes in the magnitude of the agonist burst were accomplished without changes in burst duration.

\section{Antagonist timing}

The antagonist burst in ECRL and ECU was initiated prior to the termination of the agonist burst (Fig. 2). The antagonist burst in ECU began an average of $7 \mathrm{msec}$ after the onset of movements in the radial direction (range $=0-15 \mathrm{msec}$ ) and that in ECRL began an average of $25 \mathrm{msec}$ after movements in the ulnar direction (range $=11-47 \mathrm{msec}$ ). The average duration of the antagonist bursts in ECRL and ECU was $98 \mathrm{msec}$, or nearly the same as when these muscles acted as agonists.

In most instances, the onset and duration of the antagonist burst did not vary for different amplitude movements. However, in 2 subjects, the onset of the antagonist burst for small movements $\left(5^{\circ}\right)$ was approximately $24 \mathrm{msec}$ earlier than the onset of the burst for large-amplitude movements $\left(25^{\circ}\right)$. In both subjects, the increase in burst onset occurred only for movements in 1 of the 2 directions. Thus, when step-tracking movements of the wrist were performed as fast as possible, the amplitude of the movement was varied primarily by changing the magnitude, but not the timing, of both the agonist and antagonist bursts.

The initiation of the antagonist burst occurred at least 55 msec before the peak of the initial trajectory and at least 35 msec before the initial peak of velocity. We believe that these time delays are large enough for the force generated by the antagonist burst to influence the magnitude and duration of the 
Figure 4. Kinematics and muscle activity for step-tracking movements performed at different intended speeds. Solid lines, Averages of 56 movements performed as fast as possible; dot-dashed lines, averages of 42 movements performed at natural speed; dashed lines, averages of 45 movements performed at half natural speed. The subject performed movements from an initial position of $10^{\circ}$ ulnar deviation to a target that required a change in wrist angle of $10^{\circ}$ in the radial direction. $A$ and $B$, Displacement and acceleration for these movements have been modified from Figure 6 in Hoffman and Strick (1986b). $C$, Agonist muscle activity was recorded from extensor carpi radialis longus. $D$. Antagonist muscle activity was recorded from extensor carpi ulnaris. Note that there were marked changes in the magnitude of the initial agonist and antagonist bursts of muscle activity. However, the time course of these initial bursts remained relatively constant.
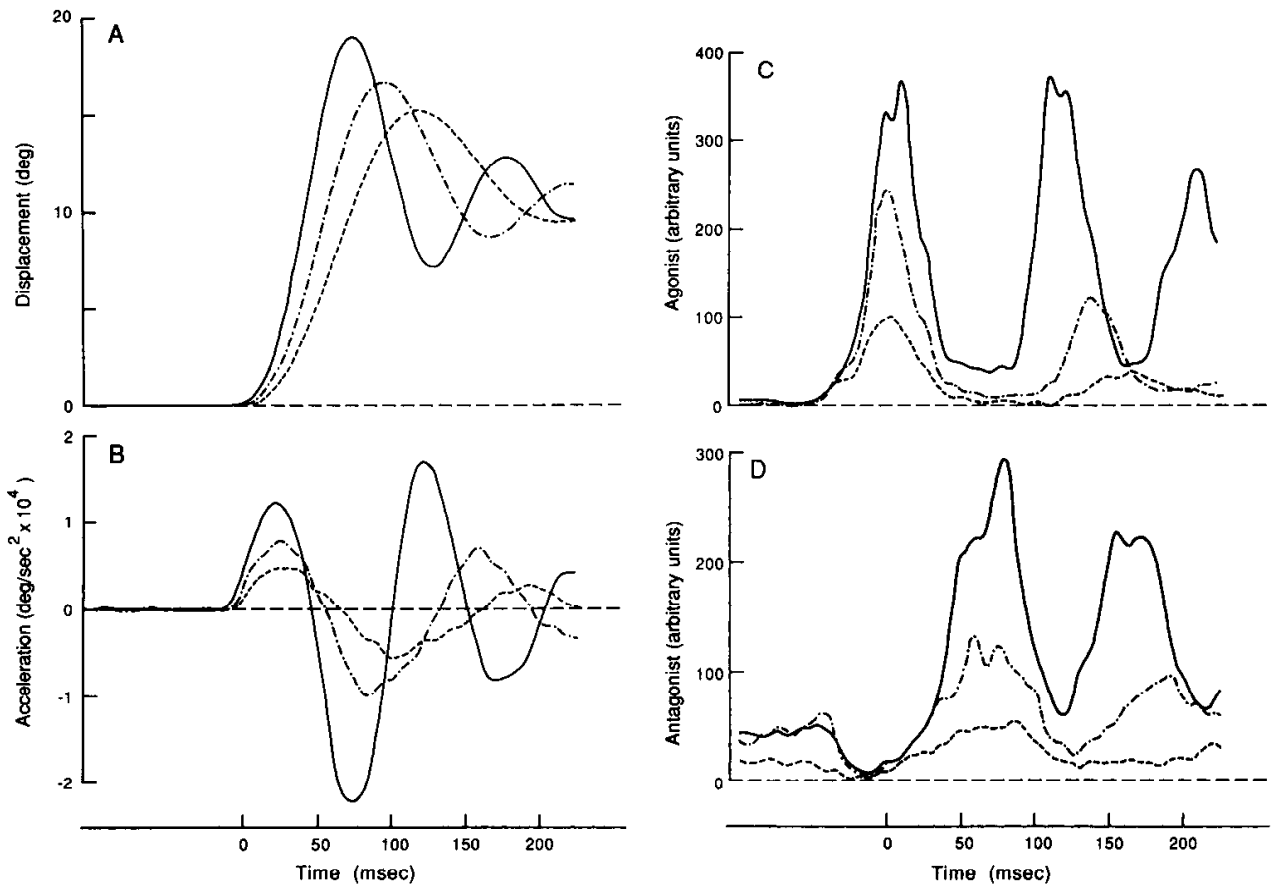

initial phase of displacement and velocity. In contrast, the initiation of the antagonist burst occurred only about $15 \mathrm{msec}$ before the initial peak of acceleration. Thus, the onset of the antagonist burst does not appear to be early enough to substantially influence the initial peaks of either acceleration or jerk in step-tracking movements.

\section{Movements performed at different intended speeds}

We used 2 different experimental paradigms to study the influence of changes in intended speed on the patterns of agonist and antagonist muscle activity. In the first paradigm, subjects were given 1 of 3 different speed instructions: "move as fast as possible," "move at your natural speed," or "move at half of your natural speed" (Figs. 4, 5). In the second paradigm, one subject was re-examined while the speed instruction was varied from trial to trial in a pseudo-random manner (Figs. 6-8). We found that subjects displayed the same alternating pattern of agonist-antagonist muscle activity for slow and fast movements (Fig. 4). However, a small percentage (28\%) of the slowest movements in one subject did not have an initial antagonist burst. These movements had durations between 145 and $218 \mathrm{msec}$ and were excluded from further study.

\section{Agonist amplitude}

When movements were made following a single speed instruction, the area of the agonist burst was linearly related to the initial peak of displacement. However, the slopes for this relation were dependent on the speed instruction and differed significantly for each instruction (e.g., Fig. 5A). Movements performed as fast as possible had the steepest slope, and movements performed at half natural speed had the shallowest slope. As a result, the agonist burst for a small movement performed as fast as possible (e.g., filled triangle in Fig. $5 \mathrm{~A}$ ) could be as large as or larger than the agonist burst for a large-amplitude movement performed at a slower intended speed (e.g., open circle and open square in Fig. 5A; see also Fig. 8). Thus, when intended speed is varied, the magnitude of the agonist burst is not uniquely related to the peak displacement of step-tracking movements.

We also examined the relations between the area of the agonist burst and the peaks of acceleration and jerk. We were interested in these kinematic variables because, as noted above, the peaks of acceleration and jerk occur early enough to be uninfluenced by the antagonist burst. The magnitude of the agonist burst was highly correlated to the initial peaks of jerk and acceleration. Furthermore, the data for movements to different targets following the 3 speed instructions were well fit by a single regression line [e.g., Fig. $5 B$, jerk: $r=0.90$; acceleration (not illustrated): $r=0.92]$. We observed similar results when a subject performed step-tracking movements to different targets and the speed instruction was randomly varied (Fig. 6). In this case, the area of the agonist burst was not well related to peak displacement (Fig. $6 A$ ). Instead, the area of the burst was highly correlated with the initial peaks of jerk (Fig. $6 B, r=0.85)$ and acceleration $(r$ $=0.86$ ). These observations indicate that when both the speed instruction and the target location are varied, the magnitude of the agonist burst is accurately reflected by the initial peaks of acceleration and jerk.

\section{Antagonist amplitude}

When subjects performed movements to different targets following a single speed instruction, there were only small changes in the magnitude of the antagonist burst. For example, the area of the antagonist burst for "natural speed" movements to the $20^{\circ}$ target was, at most, only 1.7 times greater than that of movements with the same speed instruction to the $10^{\circ}$ target (e.g., Fig. 5C, $1.3 \times$ change). No significant correlations were observed in any subject between the area of the antagonist burst and the initial peaks of displacement and its derivatives when movements were performed to different targets at "half natural speed" (e.g., Fig. 5C). Furthermore, when the speed instruction was randomly varied, the correlations between the area of the antagonist burst and the peaks of displacement (Fig. 7A) and 

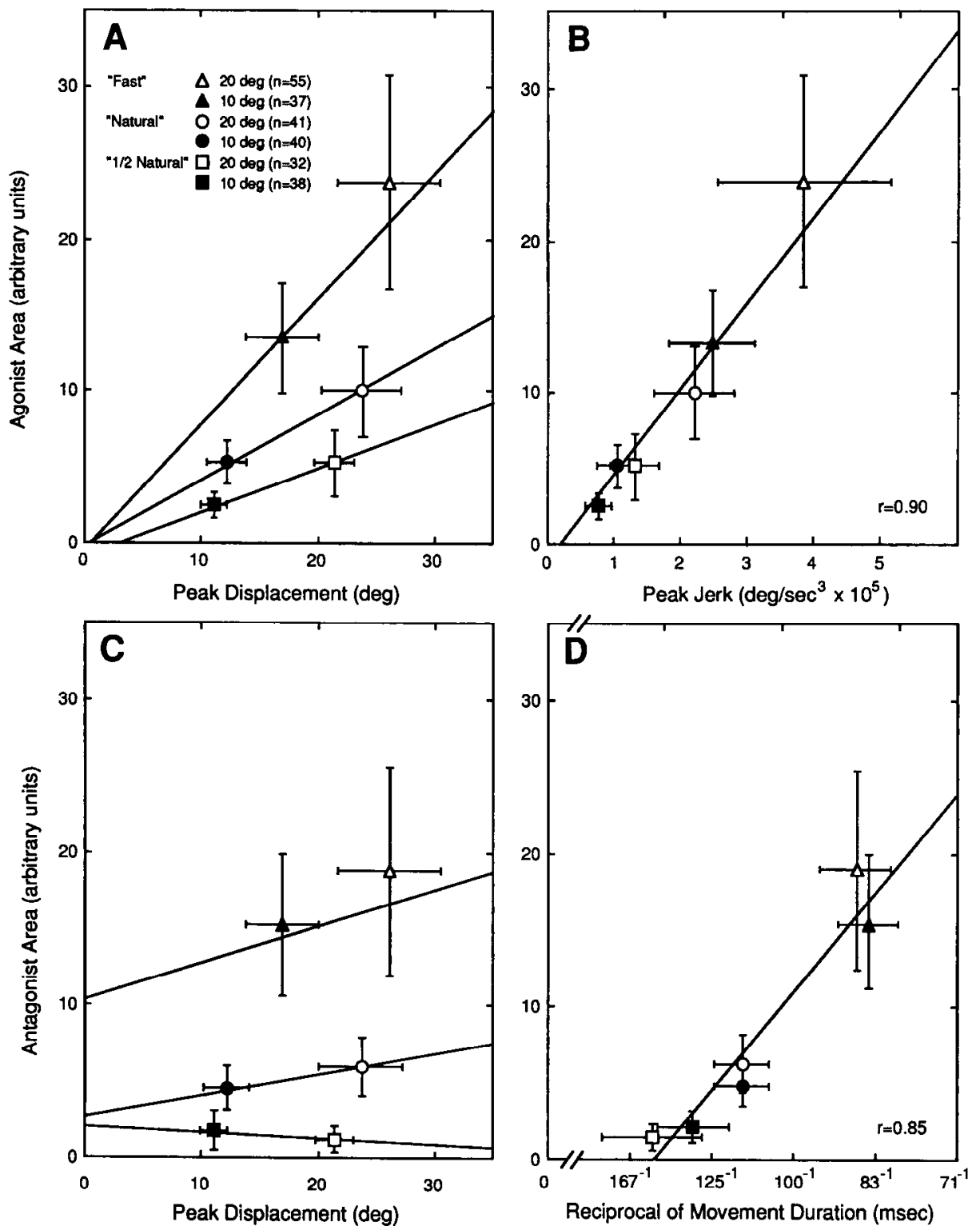

Figure 5. Relationships between kinematic parameters and the initial agonist and antagonist bursts of muscle activity for movements performed at different intended speeds. The data are from individual trials of one subject. The means and SDs are plotted for movements to targets that required $10^{\circ}$ or $20^{\circ}$ changes in wrist angle in the radial direction. The linear regression lines are plotted for the same movements, except for those performed as fast as possible. Those linear regression lines include movements with either $5^{\circ}, 15^{\circ}$, $25^{\circ}$, or $30^{\circ}$ changes in wrist angle in the radial direction. Agonist muscle activity $(A, B)$ was recorded from extensor carpi radialis longus. The correlation coefficients in $A$ are $r=0.81$ (fast), 0.83 (natural), and 0.73 (half natural). Antagonist muscle activity $(C, D)$ was recorded from extensor carpi ulnaris. The correlation coefficients in $C$ are $r=0.37$ (fast), 0.50 (natural), and -0.23 (half natural). its derivatives were weak, e.g., $r$ [displacement $]=0.11 ; r$ [velocity] $=0.33 ; r$ acceleration $]=0.43$ (compare with Fig. $7 B$, where $r[1 /$ movement duration $]=0.81)$.

In contrast, large alterations in the magnitude of the antagonist burst were associated with changes in the speed instruction (e.g., Fig. 4). For example, the magnitude of the antagonist burst when one subject performed movements "as fast as possible" to the $20^{\circ}$ target was 19.7 times larger than that for movements performed at "half natural speed" to the same target (Fig. $5 \mathrm{C}$ ). Also, large variations in the area of the antagonist burst were observed when the speed instruction was randomly varied (Fig. 7, $A, B)$.

Changes in the speed instruction resulted in large alterations in movement duration (see figure 7B in Hoffman and Strick, 1986b). Therefore, we examined the correlations between the magnitude of the antagonist burst and the duration of steptracking movements when subjects performed movements of different amplitude and intended speed. Under these conditions, we found that the area of the antagonist burst was highly cor- related with the reciprocal of movement duration $(r=0.81-$ 0.89 ). This result was observed when intended speed was altered either by giving 3 different speed instructions (e.g., Fig. $5 D$ ) or by randomly varying the speed instruction (Fig. $7 B$ ). For the data illustrated in Figure $7 B$, we also examined the correlations between the area of the antagonist burst and the product of a kinematic variable (i.e., the peaks of displacement, velocity, acceleration, or jerk) with the reciprocal of movement duration. These correlations $(r=0.52-0.65)$ were all significantly lower than that observed between the area of the antagonist burst and the reciprocal of movement duration alone $(r=0.81)$. These results indicate that, although the antagonist burst may be influenced by displacement and its derivatives, the magnitude of the burst has its most significant relationship with a variable related to the duration of a step-tracking movement.

\section{Dissociation of agonist and antagonist amplitudes}

In some circumstances, the magnitudes of the agonist and antagonist bursts appeared to covary. For example, both bursts 
Figure 6. Relationships between kinematic parameters and the initial agonist burst for step-tracking movements of varying amplitudes and intended speeds. Intended speed was randomly varied by giving speed instructions to the subject prior to each trial (see Materials and Mcthods for dctails). Agonist activity was recorded from extensor carpi radialis longus. The data plotted in both $A$ (vs the initial peak displacement) and $B$ (vs the initial peak of jerk) are from the same trials.
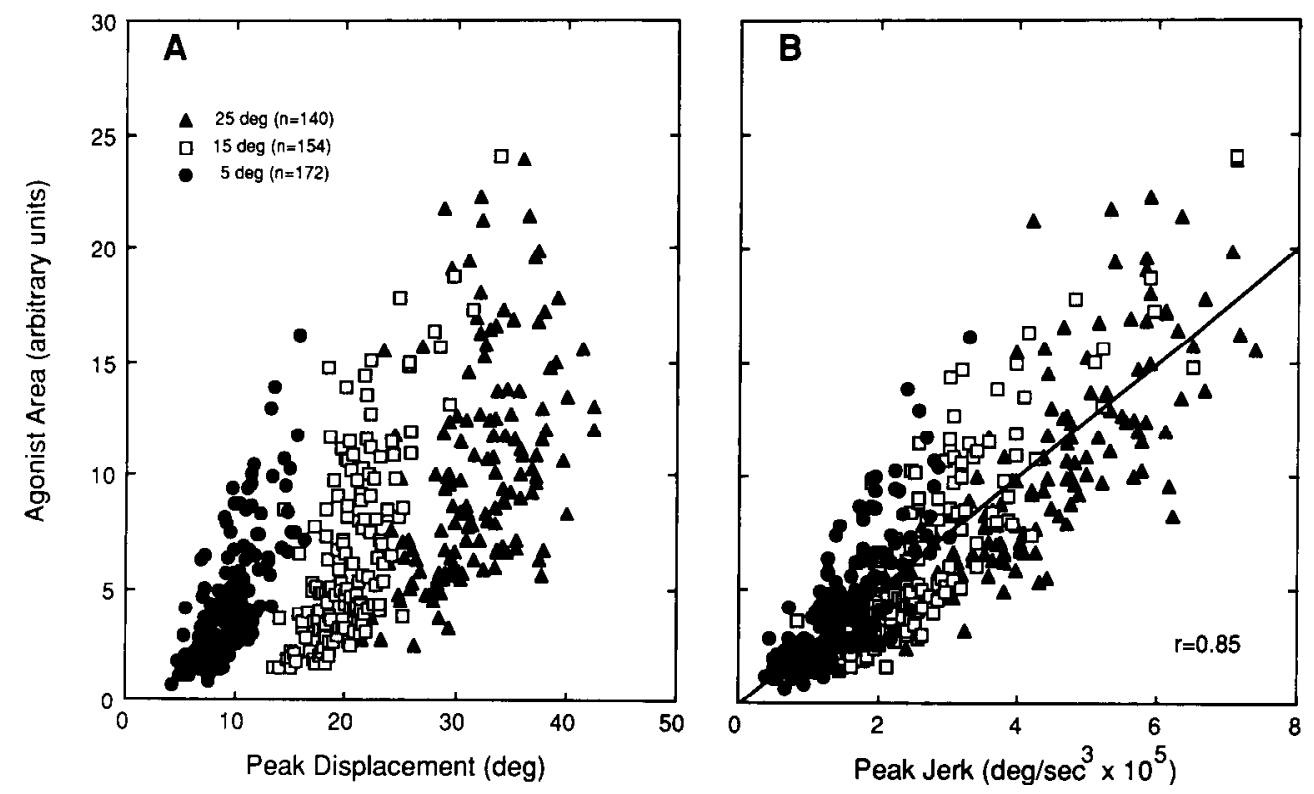

increased in size as subjects performed movements to the same target at greater intended speeds (Fig. 4). However, there were a number of clear examples where changes in the antagonist burst were dissociated from changes in the agonist burst. This result is perhaps best illustrated by examining the antagonist bursts of those movements which differed in amplitude and intended speed but were initiated by comparable agonist bursts. For example, some fast movements to the $5^{\circ}$ target and some slow movements to the $25^{\circ}$ target had agonist bursts that were nearly the same magnitude (Fig. 8). The initial increases in jerk were identical for these movements (Fig. 8, see also Fig. 6B). However, the antagonist bursts for the small, fast movements were markedly greater than those for the large, slow movements. This difference in the antagonist bursts resulted in clear alterations in the time course of movement after the initial peak of jerk. The larger antagonist burst produced the smaller-amplitude movement by shortening the initial positive phase of jerk. Based on these observations, we conclude that the processes which determine the magnitude of the antagonist burst are independent from those which generate the agonist burst. Furthermore, these results indicate that the antagonist burst can play an important role in determining the amplitude of steptracking movements.

\section{Agonist and antagonist timing}

The agonist burst in ECRL began an average of $47 \mathrm{msec}$ prior to the onset of movements performed at "natural" speed (range $=36.5-55.5 \mathrm{msec}$ ) and $50 \mathrm{msec}$ prior to the onset of movements performed at "half natural" speed (range $=36-60.5 \mathrm{msec}$ ). This burst averaged $88 \mathrm{msec}$ in duration for movements performed at "natural" speed (range $=85-91 \mathrm{msec}$ ) and $85 \mathrm{msec}$ in duration for movements performed at "half natural" speed (range $=79.5-93.5 \mathrm{msec}$ ). Thus, substantial changes in the onset and duration of the agonist burst in ECRL did not occur when subjects made movements at different intended speeds.

The timing of the antagonist burst in ECU also did not change substantially for the same movements (e.g., Fig. 4). This burst began an average of $20 \mathrm{msec}$ after the onset of movements performed at "natural" speed (range $=8-29 \mathrm{msec}$ ) and $21 \mathrm{msec}$ after the onset of movements performed at "half natural" speed (range $=9.5-27.5 \mathrm{msec}$ ). The antagonist burst in ECU averaged $98 \mathrm{msec}$ in duration for movements performed at "natural" speed (range $=91-104 \mathrm{msec}$ ) and $104 \mathrm{msec}$ in duration for movements performed at "half natural" speed (range $=85-132$ $\mathrm{msec}$ ). The relatively large range in the duration of the antagonist burst for movements performed at "half natural" speed may actually reflect the difficulty in measuring these bursts, since they were small and variable. Thus, under our experimental conditions, differences in both the amplitude and the intended speed of step-tracking movements were produced largely by altering the amplitude, and not the timing, of the agonist and antagonist bursts.

\section{Discussion}

In a prior study, we proposed that step-tracking movements of different amplitude and intended speed are generated by controlling only 2 kinematic variables (Hoffman and Strick, 1986b). One of these variables is the magnitude and the other is the duration of a derivative of displacement. In the present study we observed that the magnitude of a derivative of displacement was highly correlated with the amplitude of the agonist burst. In contrast, the duration of a derivative of displacement was inversely correlated with the amplitude of the antagonist burst. On the basis of these observations, we propose that the nervous system controls each kinematic parameter by modulating a distinct burst of muscle activity.

\section{The initial agonist burst}

In general, our results correspond to those of prior studies which examined agonist muscle activity during step-tracking movements at proximal and distal joints (e.g., Hallett and Marsden, 1979; Lestienne, 1979; Brown and Cooke, 1981; Marsden et al., 1983; Gottlieb et al., 1989a), as well as during the generation of isometric forces (e.g., Ghez and Gordon, 1987). Most investigators agree that when the speed instruction is kept constant, larger agonist bursts produce larger-amplitude movements. Also, when movement amplitude is kept constant, larger agonist bursts produce faster movements. These obscrvations 


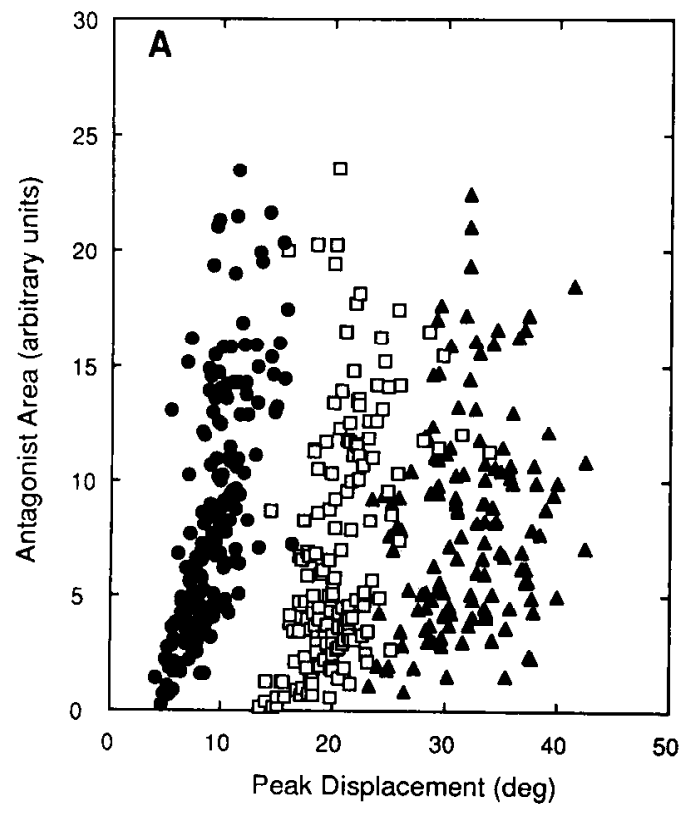

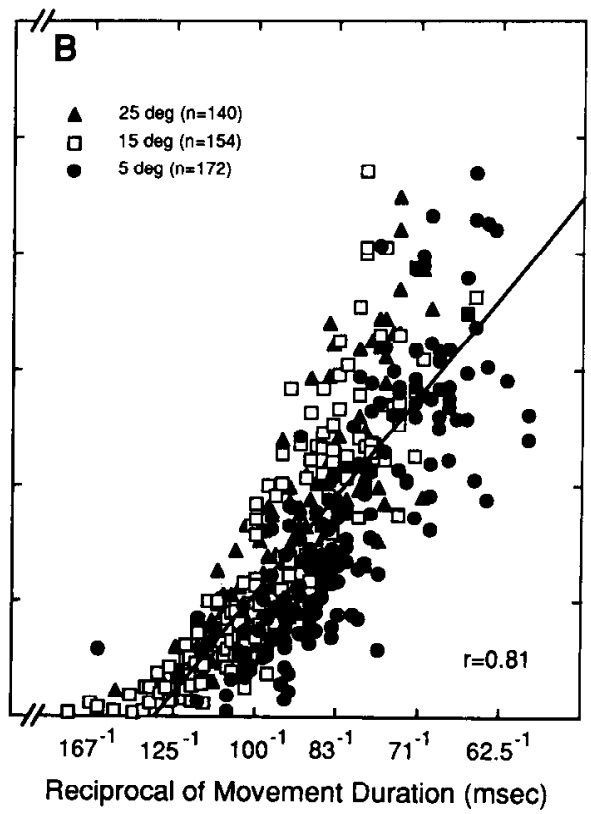

Figure 7. Relationships between kinematic parameters and the initial antagonist burst for step-tracking movements of varying amplitudes and intended speeds. Antagonist activity was recorded from extensor carpi ulnaris. The data plotted in both $A$ (vs peak displacement) and $B$ (vs the reciprocal of movement duration) are from the same trials. The agonist activity for these trials was illustrated in Figure 6. can be viewed as suggesting that the nervous system is controlling and monitoring multiple kinematic and/or dynamic variables when it adjusts the agonist burst to generate steptracking movements of different amplitude and intended speed. Our perspective differs from others because we suggest that the nervous system simplifies the generation of these movements by modulating the agonist burst in relation to a single kinematic variable.

The high correlation between the agonist burst and a derivative of displacement also suggests that a single kinematic variable (e.g., peak acceleration) reflects the magnitude of the agonist burst with remarkable accuracy. Since the mass of the limb did not change during the movements performed by our subjects, the net peak force producing joint rotation also should be highly correlated to the agonist burst. Thus, peripheral feedback about one variable (kinematic or dynamic), without reference to the target location or the speed instruction, could be used to monitor the agonist burst. In fact, a relatively pure indication of the effect of the agonist burst can only be derived by monitoring acceleration, force, or the derivatives of these variables. This is because activity in antagonist muscles occurs early enough in the movements we have examined to markedly influence the peaks of displacement and velocity.

\section{The initial antagonist burst}

The modulations in the magnitude of the antagonist burst during step-tracking movements appear paradoxical. We and others have observed that the burst varied greatly for movements performed to one target at different intended speeds (e.g., Lestienne, 1979; Brown and Cooke, 1981; Waters and Strick, 1981; Marsden et al., 1983; Karst and Hasan, 1987; Corcos et al., 1989). However, the burst varied little for different amplitude movements performed at a single intended speed (e.g., Hallett et al., 1975; Hallett and Marsden, 1979; Lestienne, 1979; Wadman et al., 1979; Brown and Cooke, 1981; Marsden et al., 1983; Gordon and Ghez, 1987; Gottlieb et al., 1989b) . In fact, in some cases large-amplitude movements were associated with a reduction in the magnitude of the antagonist burst (Marsden et al., 1983; Flament et al., 1984). Thus, even though velocity is altered for movements of different amplitude and intended speed, large alterations in the antagonist burst occurred only when the speed instruction was changed.

This paradox may be resolved by relating the magnitude of the antagonist burst to another variable, movement duration. We observed that the magnitude of the initial antagonist burst was highly correlated to the reciprocal of this variable when the amplitude and intended speed of movements were varied. We did not examine the durations of either acceleration or jerk, but we expect that the magnitude of the antagonist burst also would be highly correlated to the reciprocal of these variables. Thus, we propose that the nervous system grades the magnitude of the antagonist burst to control the duration of a derivative of displacement. This conclusion, based on step-tracking movements of the wrist, supports similar ones proposed by Wierzbicka et al. (1986), based on simulation of elbow movements, and by Gordon and Ghez (1987), based on studies of isometric movements of the elbow.

The antagonist burst may be particularly important for controlling the duration of a derivative of displacement during the fastest step-tracking movements. The minimum durations of the initial trajectories for the wrist movements of our subjects were between 58 and $71 \mathrm{mscc}$. In contrast, individual motor units in human hand muscles have twitch contraction times as long as $100 \mathrm{msec}$ (Burke, 1981). If the agonist burst was not quickly followed by an antagonist burst, then the force produced by the agonist muscle would outlast the duration of the most rapid movements. Thus, we believe that an important function of the antagonist burst during step-tracking movements is to brake the force produced by the agonist burst. In this way, the antagonist burst allows movements to be performed at speeds that are faster than would be possible if the agonist were to function in isolation (see also Wierzbicka et al., 1986; Ghez and Gordon, 1987).

It is somewhat surprising that the magnitude of the antagonist burst was not better related to movement amplitude and deceleration (see also Hallett and Marsden, 1979; Wadman et al., 1979; Brown and Cooke, 1981). Larger movements have greater accelerations and decelerations than smaller movements per- 

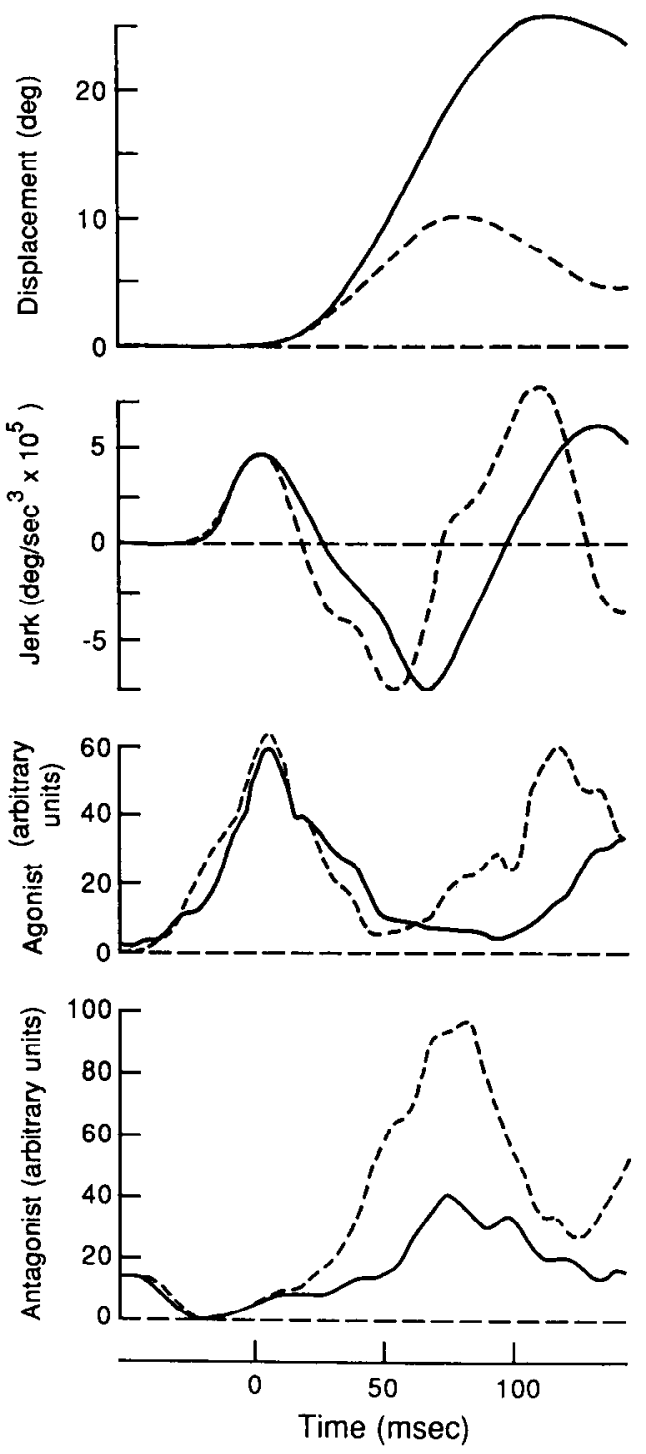

Figure 8. Averages of kinematics and muscle activity for trials with similar initial peaks of jerk. Trials were selected from data illustrated in Figures 6 and 7. Solid lines, averages of "slow" movements to the $25^{\circ}$ target $(n=21)$; dashed lines, averages of "fast" movements to the $5^{\circ}$ target $(n=21)$. Displacement and jerk for these movements have been modified from Figure 10 in Hoffman and Strick (1986b). Note that movements with the same initial peaks of jerk have similar initial agonist bursts but very different initial antagonist bursts.

formed at the same intended speed (e.g., Fig. 2). As a result, larger movements must require more "braking" force (e.g., Lestienne, 1979; Karst and Hasan, 1987) and would be expected to have larger antagonist bursts than smaller movements. Two factors might explain the weak modulation of the antagonist burst with changes in movement amplitude. First, passive viscoelastic properties of the wrist joint are likely to provide some of the additional braking force required to terminate larger steptracking movements (Lestienne, 1979). Another factor is the influence of muscle stretch on force production in the antagonist muscle. The velocity of stretch applied to the antagonist muscle was greater for larger-than for smaller-amplitude movements. When muscle activation is constant, muscle force appears to be increased in proportion to the rate at which the muscle is stretched (e.g., Komi, 1973; Zahalak et al., 1976; Heckathorne and Chil- dress, 1981). Therefore, an antagonist burst of a given amplitude would produce relatively more braking force during larger-amplitude movements than during smaller ones. In fact, marked grading of the antagonist burst for different amplitude movements may be unnecessary because the nonlinear properties of active muscle can supply the major component of the additional braking force necessary to accurately terminate larger movements.

Recently, Karst and Hasan (1987) tested the hypothesis that the antagonist burst is proportional to the torque necessary for braking movement, where braking torque $=I V^{2} / A(I=$ moment of inertia, $V=$ peak angular velocity, and $A=$ amplitude of movement). Our kinematic analysis of step-tracking movements of the wrist indicated that $A$ is highly correlated with $V$ * $T$, where $T=$ movement duration (see figure 8B in Hoffman and Strick, 1986b). When $V * T$ is substituted for $A$ in their expression, braking torque $=I V / T$. We kept $I$ constant in our experiments. Therefore, the hypothesis of Karst and Hasan (1987) predicts that in our study the area of the antagonist burst should be highly correlated with $V / T$. In fact, we observed that, for movements of different amplitude and intended speed, the antagonist burst was highly correlated with a single kinematic variable, $1 / T$. We did not perform an extensive examination of the correlations between the antagonist burst and combinations of kinematic variables. However, in one subject, we found that the correlation between the antagonist burst and $1 / T$ was higher than the correlation between this burst and $V / T$. At this point, our results emphasize the significant relation between the antagonist burst and movement duration. The potential contributions of other factors in determining antagonist activity remain to be more fully explored.

\section{Timing of the agonist and antagonist bursts}

Our results differ from those of some prior studies in that we did not observe consistent changes in the timing of the agonist burst. Movements of different amplitude and intended speed were accomplished largely by modifying the peak of this burst of muscle activity. In contrast, some, but not all, prior studies of step-tracking movements, reported modifications in the duration, as well as the peak of the agonist burst (Wadman et al., 1979; Berardelli et al., 1984; Brown and Cooke, 1984; Benecke et al., 1985; Mustard and Lee, 1987; Gottlieb et al., 1989b). Based on recent experiments and an extensive review of the literature, Gottlieb et al. (1989a; see also Corcos et al., 1989; Gottlieb et al., 1989b) concluded that the "strategy" a subject adopts to perform a movement ("speed-sensitive" or "speedinsensitive") determines the time course of agonist activity. According to this view, our subjects did not vary agonist duration because they adopted a constant strategy ("speed-sensitive") to perform movements of different amplitude and intended speed.

The strategy used to perform a task, however, may not be the only factor which determines the duration of the agonist burst. Others have observed substantial increases in burst duration in 2 conditions: (1) large-amplitude movements and (2) movements performed against external loads (e.g., Wadman et al., 1979; Berardelli et al., 1984; Brown and Cooke, 1984; Benecke et al., 1985; Mustard and Lee, 1987; Hoffman and Strick, 1989). One interpretation of these results is that when force output cannot be augmented by increasing the amplitude of the agonist burst (i.e., by recruiting additional motor units and/or modulating the frequency of those recruited), then additional force is generated by prolonging the duration of the burst (Berardelli et 
al., 1984; Benecke et al., 1985; Cheron and Godaux, 1986; Hoffman and Strick, 1989). According to this view, our subjects did not need to increase the duration of their agonist bursts to generate the forces required by our tasks. This conclusion is supported by preliminary studies in which we observed modulations in the duration of the agonist burst when we applied viscoelastic loads during step-tracking movements of the wrist (Hoffman and Strick, 1989). Thus, we believe that differences in force requirements explain some of the variation among studies in the results on the modulation of the agonist burst.

Our results also differ from those of some prior studies which reported changes in the timing of the antagonist burst. These studies observed that the antagonist burst was initiated later for larger-amplitude movements, as well as for movements performed at slower intended speeds (Wach holder and Altenburger, 1926; Lestienne, 1979; Wadman et al., 1979; Brown and Cooke, 1981; Marsden et al., 1983; Benecke et al., 1985; Mustard and Lee, 1987; Gottlieb et al., 1989b). Furthermore, the antagonist burst appeared to be delayed for movements in which the agonist burst was prolonged (e.g., Lestienne, 1979; Wadman et al., 1979; Benecke et al., 1985; Mustard and Lee, 1987; Gottlieb et al., 1989b). Since the duration of the agonist burst was kept relatively constant by our subjects, it is not surprising that we did not observe changes in the time of onset of the antagonist burst. In our preliminary experiments noted above, the antagonist burst was reduced and delayed when the duration of the agonist burst was increased (Hoffman and Strick, 1989). The factors which influence the timing of the agonist and antagonist bursts should be explored further in future experiments. However, at this point, it appears that the prolongation of the agonist burst and the delay in the onset of the antagonist burst may share common mechanisms.

\section{Central versus peripheral control of muscle activity}

There has been considerable controversy about the origin of the antagonist burst. Based on experiments in trained animals, some authors have argued that the burst is generated by reflex mechanisms which are triggered by stretch to the antagonist muscle (e.g., Terzuolo et al., 1973, 1974; Ghez and Martin, 1982). However, the antagonist activity generated by the experimental paradigms employed in these studies is unlikely to have the same origin as the initial antagonist burst examined in human experiments. Others have concluded that the antagonist burst is generated largely by central mechanisms and functions as a "preprogrammed brake" (e.g., Wachholder and Altenburger, 1926; Hallett et al., 1975). Three pieces of evidence have been used to support the concept of central generation. First, the antagonist burst begins at or just after movement onset, i.e., at times that are too early for peripheral feedback to be effective (e.g., Wachholder and Altenburger, 1926; Hallett et al., 1975). Second, the antagonist burst is present during step-tracking movements, even in subjects that lack or have reduced peripheral feedback (Hallett et al., 1975; Rothwell et al., 1982; Forget and Lamarre, 1983; Sanes and Jennings, 1984). Third, the antagonist burst is highly dependent upon central programming, for it is present when subjects intend to accurately terminate movements and absent when movements are terminated by a mechanical stop (Waters and Strick, 1981; Marsden et al., 1983; Benecke et al., 1985).

The present experiments provide additional support for the concept that the antagonist burst is generated via central mechanisms. We found that the antagonist burst was graded in re- lation to movement duration. Movement duration depended on the speed instruction given to the subject. Therefore, the antagonist burst varied in relation to a central command, i.e., the subject's intent to move at a specific speed. We also found that, for some movements, a subject could have agonist bursts of identical magnitude and yet have antagonist bursts that differed considerably (Fig. 8). Thus, the magnitude of the antagonist burst, in some cases, was clearly dissociated from the magnitudes of both the initial agonist burst and the initial stretch to the antagonist produced by agonist activity (see also Ghez and Gordon, 1987, for similar observations during isometric movements). These dissociations strongly imply that the antagonist burst is centrally generated. Furthermore, our observations suggest that the burst is generated by central mechanisms which are, in part, separate from those which control the agonist burst. It should be noted, however, that the evidence cited above for the central generation of the antagonist burst does not preclude the possibility that peripheral feedback could modify antagonist muscle activity during normal step-tracking movements (e.g., Hallett and Marsden, 1979).

\section{Control of the initial movement trajectory by coordinated bursts of muscle activity}

Although we have emphasized that there is independent control of the magnitudes of the initial agonist and antagonist bursts, it is also important to recognize that the magnitude and timing of these 2 bursts of muscle activity must be precisely coordinated. For example, we noted above that identical agonist bursts could generate movements of very different amplitude and intended speed. This was only possible because the antagonist bursts for the 2 movements were significantly different (Fig. 8). This observation indicates that the antagonist, by acting to brake the force generated by the agonist, helps to specify not only the duration, hut also the ultimate displacement of step-tracking movements. Thus, the accuracy of step-tracking movements depends upon the precise coordination of the 2 bursts of muscle activity. We believe that a challenge for the future is to determine the neural structures which participate in the independent generation and/or coordinated control of the 2 bursts of muscle activity.

\section{References}

Benecke, R., H.-M. Meinck, and B. Conrad (1985) Rapid goal-directed elbow flexion movements: Limitations of the speed control system due to neural constraints. Exp. Brain Res. 59: 470-477.

Berardelli, A., J. C. Rothwell, B. L. Day, T. Kachi, and C. D. Marsden (1984) Duration of the first agonist EMG burst in ballistic arm movements. Brain Res. 340: 183-187.

Brown, S. H. C., and J. D. Cooke (1981) Amplitude- and instructiondependent modulation of movement-related electromyogram activity in humans. J. Physiol. (Lond.) 316: 97-107.

Brown, S. H., and J. D. Cooke (1984) Initial agonist burst duration depends on movement amplitude. Exp. Brain Res. 55: 523-527.

Burke, R. E. (1981) Motor units: Anatomy, physiology and functional organization. In Handbook of Physiology, Sect. 1: The Nervous System, Vol. 2: Motor Control, V. B. Brooks, ed., pp. 345-422, American Physiological Society, Bethesda, MD.

Cheron, G., and E. Godaux (1986) Self-terminated fast movement of the forearm in man: Amplitude dependence of the triple burst pattern. J. Biophys. Biomech. 10: 109-117.

Corcos, D. M., G. L. Gottlieb, and G. C. Agarwal (1989) Organizing principles for single joint movements: II. A speed-sensitive strategy. J. Neurophysiol, 62: 358-368.

Flament, D., J. Hore, and T. Vilis (1984) Braking of fast and accurate elbow flexions in the monkey. J. Physiol. (Lond.) 349: 195-203.

Forget, K., and Y. Lamarre (1983) Antagonist muscle activity during 
rapid flexion of the forearm in a functionally deafferented patient. Soc. Neurosci. Abstr. 9: 630.

Ghez, C., and J. Gordon (1987) Trajectory control in targeted force impulses. I. Role of opposing muscles. Exp. Brain Res. 67: 225-240.

Ghez, C., and J. H. Martin (1982) The control of rapid limb movement in the cat. III. Agonist-antagonist coupling. Exp. Brain Res. 45: $115-$ 125.

Gordon, J., and C. Ghez (1987) Trajectory control in targeted force impulses. II. Pulse height control. Exp. Brain Res. 67: 241-252.

Gottlieb, G. L., and G. C. Agarwal (1970) Filtering of electromyographic signals. Am. J. Phys. Med. 49: 142-146.

Gottlieb, G. L., D. M. Corcos, and G. C. Agarwal (1989a) Strategies for the control of voluntary movements with one mechanical degree of freedom. Behav. Brain Sci. 12: 189-250.

Gottlieb, G. L., D. M. Corcos, and G. C. Agarwal (1989b) Organizing principles for single joint movements: I. A speed-insensitive strategy. J. Neurophysiol. 62: 342-357.

Hallett, M., and C. D. Marsden (1979) Ballistic flexion movements of the human thumb. J. Physiol. (Lond.) 294: 33-50.

Hallett, M., B. T. Shahani, and R. R. Young (1975) EMG analysis of stereotyped voluntary movements in man. J. Neurol. Neurosurg. Psychiatry $38: 1154-1162$.

Heckathorne, C. W., and D. S. Childress (1981) Relationships of the surface electromyogram to the force, length, velocity and contraction rate of the cineplastic human biceps. Am. J. Phys. Med. 60: 1-19.

Hoffman, D. S., and P. L. Strick (1981) Trajectories of ballistic movement can be altered by modifying antagonist muscle activity. Soc. Neurosci. Abstr. 7: 479.

Hoffman, D. S., and P. L. Strick (1982) Evidence that antagonist muscle activity is centrally generated. Soc. Neurosci. Abstr. 8: 733.

Hoffman, D. S., and P. L. Strick (1984) Controlled variables in steptracking movements of the wrist. Soc. Neurosci. Abstr. 10: 332.

Hoffman, D. S., and P. L. Strick (1986a) Activity of wrist muscles during step-tracking movements in different directions. Brain Res. 367: 287-291.

Hoffman, D. S., and P. L. Strick (1986b) Step-tracking movements of the wrist in humans. I. Kinematic analysis. J. Neurosci. 6: 33093318.

Hoffman, D. S., and P. L. Strick (1989) Force requirements and patterns of muscle activity. Behav. Brain Sci. 12: 221-224.

Karst, G. M., and Z. Hasan (1987) Antagonist muscle activity during human forearm movements under varying kinematic and loading conditions. Exp. Brain Res. 67: 391-401.

Komi, P. V. (1973) Measurement of the force-velocity relationship in human muscle under concentric and eccentric contractions. In Medicine and Sport. Vol. 8: Biomechanics III, pp. 224-229, Karger, Basel.

Lestienne, F. (1979) Effects of inertial load and velocity on the braking process of voluntary limb movements. Exp. Brain Res. 35: 407-418.

Marsden, C. D., J. A. Obeso, and J. C. Rothwell (1983) The function of the antagonist muscle during fast limb movements in man. J. Physiol. (Lond.) 335: 1-13.

Mustard, B. E., and R. G. Lee (1987) Relationship between EMG patterns and kinematic properties for flexion movements at the human wrist. Exp. Brain Res. 66: 247-256.

Rothwell, J. C., M. M. Traub, B. L. Day, J. A. Obeso, P. K. Thomas, and C. D. Marsden (1982) Manual motor performance in a deafferented man. Brain 105: 515-542.

Sanes, J. N., and V. A. Jennings (1984) Centrally programmed patterns of muscle activity in voluntary motor behavior of humans. Exp. Brain Res. 54: 23-32.

Terzuolo, C. A., J. F. Soechting, and P. Viviani (1973) Studies on the control of some simple motor tasks. I. Relations between parameters of movements and EMG activities. Brain Res. 58: 212-216.

Terzuolo, C. A., J. F. Soechting, and N. A. Ranish (1974) Studies on the control of some simple motor tasks. V. Changes in motor output following dorsal root section in squirrel monkey. Brain Res. 70: 521526.

Wachholder, K., and H. Altenburger (1926) Beitrage zur Physiologie der willkurlichen Bewegung. $X$. Einzelbewegungen. Pfluegers Arch. 214: 642-661.

Wadman, W. J., J. J. Denier van der Gon, R. H. Geuze, and C. R. Mol (1979) Control of fast goal-directed arm movements. J. Hum. Move. Stud. 5: 3-17.

Waters, P., and P. L. Strick (1981) Influence of "strategy" on muscle activity during ballistic movements. Brain Res. 207: 189-194.

Wierzbicka, M. M., A. W. Wiegner, and B. T. Shahani (1986) Role of agonist and antagonist muscles in fast arm movements in man. Exp. Brain Res. 63: 331-340.

Zahalak, G. I., J. Duffy, P. A. Stewart, H. M. Litchman, R. H. Hawley, and P. R. Paslay (1976) Partially activated human skeletal muscle: An experimental investigation of force, velocity and EMG. J. Appl. Mech. 98: 81-86. 